\title{
BIOMPHALARIA STRAMINEA NO PERU E SUA SUSCETIBILIDADE A CEPAS BRASILEIRAS DE SCHISTOSOMA MANSONI
}

\author{
C.A. Cuba Cuba*e L.R. Corrêa**
}

Em Maio de 1973, um dos autores (C.A.C.) coletou na localidade de Imacita, Província de Bagua, Departamento de Amazonas, vários espécimes de Biomphalaria straminea (Dunker, 1848), uma espécie que, até então, não havia sido assinalada no Peru.

Descendentes destes individuos foram submetidos a provas de suscetibilidade as cepas $B H$ e SJ de Schistosoma mansoni que, em condições naturais, evoluem em B. glabrata de Belo Horizonte e B. tenagophila de São José dos Campos, respectivamente.

Oitenta espécimens foram expostos à cepa BH dos quais em 13 ou 16,2\% a infecção evoluiu caracteristicamente até a formação de esporocistos secundários sem haver, contudo, eliminação de cercárias, mesmo no individuo que apresentou uma sobrevivência de 88 dias após a exposição. Não se verificou cura espontânea neste lote.

Entre as 40 B. straminea expostas à cepa SJ 9 ou 22,5\% infectaram-se, sendo que apenas duas eliminaram poucas cercárias aos 57 e 77 dias após a exposição, por dois dias consecutivos, tendo uma morrido e uma se curado espontaneamente. A cura espontânea do parasitismo foi notado em mais dois individuos, nos quais a infecção foi observada a través da concha.

Cortes histológicos seriados de 9 caramujos, expostos individualmente a 50 miracidios da cepa BH e fixados entre 6 e 120 horas após a exposição, mostraram esporocistos em desenvolvimento e esporocistos invadidos por amebócitos, sem formação de granulomas por parte do hospedeiro, fato assinalado em caramujos suscetiveis.

A população estudada comportou-se experimentalmente de modo seme/hante a outras populações de B. straminea testadas em laboratbrio, isto e, com baixa suscetibilidade, embora tal comportamento não afaste a possibilidade dela vir a manter o ciclo do parasita em sua área de distribuição.

\section{INTRODUÇÃO}

Biomphalaria straminea, importante hospedeiro intermediário de Schistosoma mansoni no Nordeste do Brasil, apresenta vasta distribuição geográfica sendo encontrada na Venezuela, Guianas, Paraguay e Brasil, alcançando perto de $20^{\circ}$ latitude sul ${ }^{11}$. Ainda no Brasil sua presença é assinalada em várias áreas da Bacia Amazônica como em Manaus ${ }^{1}$, tendo sido incriminada como vetor de esquistossomose em Fordlandia, Estado do Pará ${ }^{10}$, um foco atualmente extinto.

Em relação a seu potencial de vetor de $S$. mansoni e visando caracterizar o que foi denominado como "qualidade de vetor", Barbosa \& Coelho ${ }^{2}$ : Coelho \& Barbosa ${ }^{5}$. pesquisaram as relações hospedeiro parasita de $B$. straminea a uma cepa pernambucana de S. mansoni. Posteriormente, Barbosa \& Figueiredo $^{3}$ realizaram estudos sobre a suscetibilidade de diversas cepas de $B$. stra-

\footnotetext{
* Professor da Faculdade de Ciências da Saúde. Universidade de Brasília. Brasillia D.F., Brasil.

- Professora do Institut o de Biologia. Universidade de Brasilia. Brasília, D.F., Brasil.

Recebido para publicação em 15.6.1977
} 
minea, coletadas em 5 Estados do Nordeste brasileiro, à uma cepa de $S$. mansoni verificando, geralmente, baixas taxas de infecção experimental.

No presente trabalho são apresentados os resultados do estudo da suscetibilidade ao $S$. mansoni de B. straminea procedente do Peru, país onde até agora essa espécie não tinha sido assinalada.

\section{MATERIAL E MÉTODOS}

Nas experiências foram usadas duas cepas de Schistosoma mansoni a cepa $\mathrm{BH}$, isolada em 1959 de um paciente crônico de esquistossomose contraida em Belo Horizonte, MG., e a cepa SJ, isolada em 1962 de Biomphalaria tenagophila naturalmente infectada, coletada em São José dos Campos, Estado de São Paulo, ambas mantidas regularmente em camundongos albinos no Laboratório de Parasitologia do Instituto de Biologia, da Universidade de Brasília.

Caramujos criados no laboratório (4.5 $9 \mathrm{~mm}$ de diâmetrol descendentes de espécimens coletados na localidade de Imacita, Província de Bagua, Departamento de Amazonas, Peru, numa área denominada "selva alta", a $650 \mathrm{~m}$. sobre o nível do mar, foram individualmente expostos a $10 \mathrm{mira}$ cídios de $S$. mansoni (Cepas BH e SJ). A concentração dos miracídios era efetuada pela técnica de Chaia a partir de fezes de camundongos albinos infectados com as cepas estudadas. No estereomicroscópio, os miracídios eram coletados com a ajuda de pipeta Pasteur de calibre fino e contados, sendo depositados em placa de petri de $4 \mathrm{cms}$ de diâmetro, contendo água desclorada. Especial cuidado na escolha dos miracidios foi tomada, pois somente os mais ativ os foram empregados. Os caramujos eram colocados nas placas em contacto com os miracídios por um período de 6 hs. após o qual eram colocados em aquários balan. ceados. Diariamente observavase os espécimens e no caso da morte de algum deles procedia-se a dissecção e examinava-se a presença de formas lavárias em desenvolvimento do $S$. mansoni. Entre o $26{ }^{\circ}$ e 30 ? dias após a exposição e depois cada 48 hs. os caramujos eram isolados individualmente em pequenos vidros contendo água desclorada e expostos a luz de lâmpadas elétricas de acordo com a técnica padronizada neste laboratório.
O material destinado a estudo histológico era obtido pela morte dos caramujos infectados na água quente $\left(70^{\circ} \mathrm{C}\right)$ e retirada do corpo puxando e esticando as partes moles sobre papel de filtro. Posteriormente se fixava em Bouin (12-24 hs), sendo retirado o excesso de ácido picrico com 3 passagens em álcool h-butílico e incluídos em parafina. Cortes seriados de $6 \mathrm{u}$ da totalidade do corpo do animal foram corados pela Hema toxilina-eosina.

Desenhos em câmara clara da genitália de alguns exemplares foram realizados. Como controle para as diversas experiências de infecção foram utilizados B. glabrata de Belo Horizonte, MG.

\section{RESULTADOS}

O estudo das conchas e genitália dos moluscos coletados no Peru mostraram similitude morfológica com Biomphalaria straminea (Dunker. 1858) (Fig. 1).

A TABELA I apresenta os resultados da exposição de 80 caramujos à cepa $B H$ de $S$. mansoni, dos quais $13 \mathrm{~B}$. straminea, tornaramse infectados obtendo-se um índice de infecção de $16,2 \%$. Todavia nenhum deles eliminou cercárias, embora houvesse invasão maciça de esporocistos secundários nos órgãos internos e a evolução da infecção ter sido acompanhada durante 3 meses, aproximadamente. Très espécimens com 46, 55 e 88 dias após exposição apresentaram esporocistos secundários finos, sem contudo haver ev olução para cercárias.

A observação dos cortes histológicos revelou que a massa cefalopedal e o manto foram as áreas com maior número de esporo cistos. $\mathrm{O}$ exame do aspecto dos esporocistos revelou formas aparentemente integras (inalteradas), com numerosas células germinativas em multiplicação e presença de massa neural, sem reação tissular do hospedeiro, junto a formas em destruição (alteradas), invadidas por amebócitos e com discreta reação celular. Nos esporocistos que apresentaram alterações degenerativas não foi verificada a presença de granulomas. De 86 esporocistos observados nos cortes histológicos seriados de 9 caramujos infectados, $73,2 \%$ se apresentaram aparentemente íntegros.

Um indice de infecção de $22,5 \%$ foi observado entre os $40 \mathrm{~B}$. straminea expostos à cepa SJ (TABELA II), nove dos quais tornaram-se intensamente infectados. 


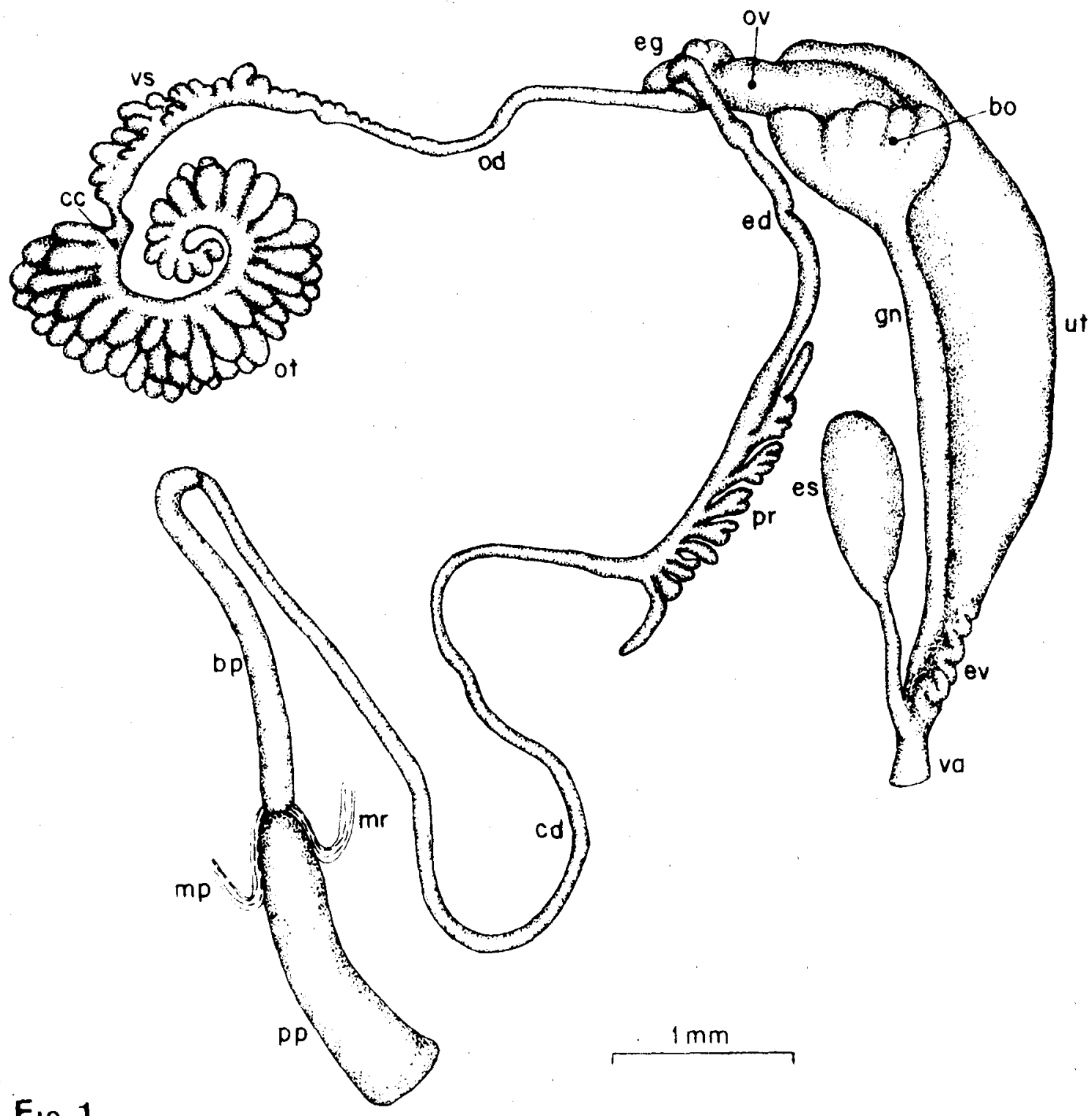

\section{Fig. 1}

Biomphalario straminea de Bagua, Peru: sistema genital bo, bolsa do oviduto; bp, bainho do pênis; cc, canal coletor do ovoteste; cd, canal deferente; ed, espermiduto, eg, encruzilhada genital; es, esper mateca; ev, enrugamento vaginal; gn, glândula nidamental; mp, múscu 10 protrator; $\mathrm{mr}$, músculo retrator; od, ovispermiduto; ot, ovoteste; ov, ovi. duto; pp, prepúcio; pr, próstała; ut, utero; va, vagina; vs, vesicula semini. 


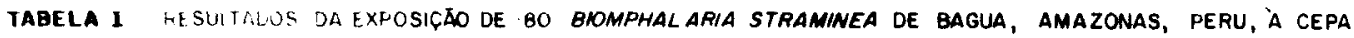
BH DE SCHISTOSOMA MANSONI ( 10 MIRACÍOIOS POR CARAMUJO).

\begin{tabular}{|c|c|c|}
\hline $\begin{array}{l}\text { DIAS APÓS } \\
\text { EXPOSICYAOO }\end{array}$ & $\begin{array}{l}\text { CARAMUJOS } \\
\text { EXAMINADOS }\end{array}$ & RESULTAOOS* * \\
\hline 320 & 12 & MORTOS NEGATIVOS \\
\hline 21 & 1 & ES NO OT, GD, CR, PN, CP \\
\hline $22-28$ & 5 & MORTOS NEGATIVOS \\
\hline 29 & । & ES NA GD, PP, MC. O RESTO AUTOLISADO \\
\hline $30-33$ & 2 & MORTOS NEGATIVOS \\
\hline 34 & 1 & ES $S^{*} O$ OT, GD, QA, PP, CR \\
\hline 35 & 1 & MORTO NEGATIVO \\
\hline 36 & 1 & ES OT, GD, CR, MC \\
\hline $37-41$ & 2 & MORTOS NEGATIVOS \\
\hline $42-43$ & 2 & | COM ES NA CR, PN, IN; | COM ES NO OT, BO, GN, CR, TR, PP, MC \\
\hline 46 & ! & ES NO OT, GD, PP \\
\hline 47 & $\because$ & ES NO OT, GD, CR, PN \\
\hline $48-50$ & 8 & MORTOS NEGATIVOS \\
\hline 51 & 1 & ES iNO OT, GD, ET, IN, MC, PP, PN \\
\hline $52 \cdot 54$ & 4 & MORTOS NEGATIVOS IES. ANTENA. PÉ. COLAR MANTO CR ON. OD MC PAREDE ESTÖMABO \\
\hline 55 & , & $E S^{*}$ NO OT, PP, MC,TR,CP \\
\hline $56 \cdot 84$ & 21 & DISSECAOOS NEGATIVOS \\
\hline 85 & ! & ES NO OT, GA, PP, CR, CM \\
\hline 38 & 1 & ES NA $^{\text {\# }}$; O RESTO AUTOLISADO \\
\hline 9096 & 3 & DISSECADOS NEGATIVOS \\
\hline
\end{tabular}

* esporotistos secundarios delgados

* incice de infeccaso $16,2 \%$

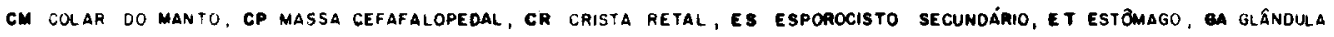
do albimen, go glânoula digestiva, in intestino, me músculo columelar, ot onoteste, pp pareoe pulmonar tr tuao renal 
TABELA II - RESULTADOS DA EXPOSICAÃO DE 40 BIOMPHALARIA STRAMINEA DE BAGUA, AMAZONAS, PERU, À CEPA SJ DE SCHISTOSOMA MANSONI ( 10 MIRACIDIOS POR CARAMUJO)

\begin{tabular}{|c|c|c|}
\hline $\begin{array}{l}\text { DIAS APOS } \\
\text { EXPOSICĀO }\end{array}$ & $\begin{array}{l}\text { CARAMUJOS } \\
\text { EXAMINADOS }\end{array}$ & RESULTAOOS* \\
\hline $15-32$ & 6 & MORTOS AUTOLISADOS NEGATIVOS \\
\hline $35-44$ & 7 & DISSECADOS NEGATIVOS \\
\hline 54 & 1 & ELIMINOU CERCÁRIA $(N \circ 1)^{* *}$ \\
\hline 57 & 2 & POSITIVOS. ES OBSERVADOS ATRAVÉS DA CONCHA $(\text { NoS } 3,4)^{*}$ \\
\hline 70 & 6 & DISSECADOS NEGATIVOS \\
\hline 73 & 1 & POSITIVO ES OBSERVADOS ATRAVÉS DA CONCHA (No 5) \\
\hline 75 & 4 & DISSECADOS NEGATIVOS \\
\hline 77 & 1 & ELIMINOU CERCÁRIAS $(N=2)^{* *}$ \\
\hline 78 & 1 & POSITIVO. ES OBSERVADOS ATRAVÉS DA CONCHA $(N=6)^{* *}$ \\
\hline 81 & 1 & ES NO OT, GD, ET, GA, PP, CR, MC, CP \\
\hline 84 & 2 & I COM ES NO OT, GD, ET, CR, PP, MC, CP; | COM ES NO OT, GD, ET, GN, TR, PP, CP \\
\hline 86 & 8 & DISSECADOS NEGATIVOS \\
\hline
\end{tabular}

* índice de infeccão $22,5 \%$ indice eliminacăo cercárias $5 \%$

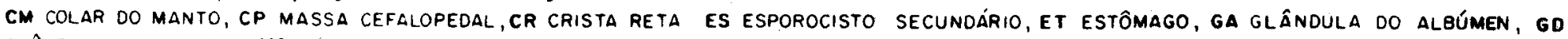
GLiNOULA NIDAMENTAL, MC MUSCULO COLUMELAR, OT OVOTESTE, PP PAREDE PULMONAR, TR TUBO RENAL 
TABELA III - ELIMINACĀO DE CERCÁRIAS E EVOUÇĀO DA INFECÇĀO EM BIOMPHALARIA STRAMINEA (PERU) INFETADAS COM A CEPA SJ DE SCHISTOSOMA MANSONI.

\begin{tabular}{|c|c|c|c|c|}
\hline $\begin{array}{l}\text { CARAMUJO } \\
\text { №*** }\end{array}$ & $\begin{array}{l}\text { PERIODO DE } \\
\text { ELIMINAÇÃO }\end{array}$ & $\begin{array}{l}\text { NQ TOTAL DE } \\
\text { CERCÁRIAS }\end{array}$ & $\begin{array}{l}\text { DIAS APÓS } \\
\text { EXPOSICĀO }\end{array}$ & OBSERVAÇŌES **** \\
\hline 1 & 2 & 9 & 119 & SACRIFICADO: CURA ESPONTÂNEA \\
\hline 2 & 2 & 10 & 79 & MORREU COM INFECCCĀO MACICAA. ES NO OT, GD, GA, CR,PP \\
\hline 3 & - & - & 65 & MORREU. ES NO OT, GO, ET,PP, CR, CP \\
\hline 4 & - & - & 106 & SACRIFICADO: CURA ESPONTÂNEA \\
\hline 5 & - & - & 84 & MORTO ACIDENTALMENTE. ES NO OT, GD, ET, PP, PN, CR, CM,CP \\
\hline 6 & - & - & 90 & SACRIFICADO: CURA ESPONTÃNEA \\
\hline
\end{tabular}

* veja a tabela i

* * contados em dias alternados

* * * Cu colar do manto, cP massa cefalopedal, cr crista retal, es esporocisto secundário, et estómago,

ea glándula do albúmen, go glándula digestiva, ot ovoteste, pP parede pulmonar, pN pNeumóstoma 
Apenas dois destes últimos eliminaram cercárias (5\% do total dos caramujos expostos). A liberação de cercárias (TABELA III) foi acompanhada nos dois caramujos $(1,2)$ sendo que ambos só eliminaram 9 e 10 cercárias respectivamente durante dois dias, após o que 0 n? 1 deixou de eliminar $e$ dissecado aos 119 dias, após exposição, não apresentou sinal de infecção, demonstrando cura da infecção. $\mathrm{O}$ n! 2 morreu no terceiro dia mostrando intensa infecção por esporo cistos secundários, do ovoteste e glândula digestiva. Os caramujos n? 4 e 6 , cuja infecção foi comprovada aos 57 e 78 dias após a exposição pela observação de esporocistos através da concha, não apresentaram mais sinal de formas larvárias quando dissecados aos 106 e 90 dias, respectivamente. Dessa forma os espécimens foram considerados curados espontaneamente da infecção.

\section{DISCUSSĀO}

Embora no Peru não tenha sido verificada a presença de Schistosoma mansoni e esquistossomose, moluscos vetores do parasita estão distribuldos em certas áreas do território peruano. Paraense $\&$ cols. ${ }^{9}$ assinalaram a espécie Biomphalaria tenagophila no vale interandino de Condebamba, Departamento de Cajamarca, a qual se mostrou altamente sucetível à infecção experimental por uma cepa brasileira de $S$. mansoni.

Neste trabalho, comunicamos pela primeira vez a presença de $B$. straminea, no Peru, outro importante vetor de esquistossomose no nordeste brasileiro, na localidade de Imacita, Província de Bagua, Departamento de Amazonas, ampliando, desta forma, o conhecimento da distribuição geográfica dessa espécie ${ }^{11}$.

Estudos experimentais com cepas de $B$. straminea e $S$. mansoni de diversas procedências têm evidenciado indices de infecção não superiores a $5 \%$, demonstrando a baixa suscetibilidade dessa espécie ao trematódeo ${ }^{2,3,7}$.

A cepa de B. straminea testada no presente trabalho apresentou índice de infecção com eliminação de cercárias de $5 \%$, quando exposta à cepa $S J$ de $S$. mansoni; este dado é comparável aos relatados pelos autores anteriormente citados. Quando testados com a cepa BH não houve eliminação de cercárias. No entanto, para se estudar a suscetibilidade de um molusco vetor, observaçōes sobre a infecção e evolução das formas intramolusco devem ser realizadas.

O comportamento da evolução da infecção nos diversos órgãos internos dos caramujos expostos ao parasita mostrou diferenças importantes entre as cepas $\mathrm{BH}$ e SJ. A cepa BH evoluiu até esporocistos secundários, invadindo ovoteste e glândula digestiva, apresentando períodos de infecção tão longos como 51 e 88 dias, com indice de $16,2 \%$ dos caramujos expostos, porém sem elimi nação de cercárias. A cepa SJ invadiu os órgãos internos de vários espécimens, completando sua evolução, com eliminação de cercárias em dois exemplares cujos períodos pré patentes de infecção foram de 54 e 77 dias, respectivamente.

Esta aparente contradição entre índice de infecção $(16,2 \%$ e $22,5 \%$ ) e indice de eliminação de cercárias $(5 \%$ e $0,0 \%$ ), se deve ao fato de ser considerado infectados todos os caramujos que apresentaram formas evolutivas de $S$. mansoni, após sua exposição à miracidios do trematódeo. O comportamento da infecção em $B$. straminea, e outras espécies de planorb/deos vetores de esquistossomose, têm sido muito bem estudados por Barbosa \& Coelho ${ }^{2}$; Paraense \& Corrêa ${ }^{8}$; Paraense \& cols. ${ }^{9}$; Corrêa \& Paraense ${ }^{6}$, em seus estudos com outros modelos experimentais.

E verdade que do ponto de vista epidemiológico, a taxa de infecção com eliminação de cercárias é a característica mais relevante em infecções experimentais de planorbideos. Entretanto, em estudos sobre suscetibilidade devem ser estabelecidas as características de evolução das formas larvárias intra-molusco. A mortalidade e a cura espontânea observadas são, evidentemente, conseqüências da relação parasitahospedeiro.

Em relação ao exame de $B$. straminea sacrificados durante as primeiras horas após infecção houve um maior porcentual de esporocistos íntegros $(73,2 \%)$, não sendo evidenciado um aumento de formas lavárias destruldas à medida que a infecção se tornava mais antiga. Este fato é discordante das observações realizadas por Coelho \& Barbosa $^{5}$ em suas experiências com $B$. straminea de Pernambuco. Esporocistos invadidos por amebócitos foram encontrados geralmente em tecidos altamente vascularizados, mas sem reação tissular intensa e nem formaçáo de granulomas. Aparentemente as 
formas larvárias evoluem e migram até o ovoteste e glâncula digestiva, mas falham no momento de diferenciar-se em cercárias e as vezes regridem até o desaparecimento total sem deixar sinais de restos parasitários. Esse fato foi observado com a cepa SJ (TABELAS $\|$ e $\|I\|$ ) e foi reportado anteriormente por Coelho \& Barbosa ${ }^{5}$.
A avaliação do possível papel epidemiolbgico de $B$. straminea na região amazố nica peruana, não pode ser determinada neste trabalho, porém as observações realizadas contribuem para o conhecimento de seu comportamento face à infecção por $S$. mansoni.

\section{SUMMARY}

In May 1973 one of the Authors (C.A.C.) collected specimens of Biomphalaria straminea (Dunker 1848) at Imacita, Bagua Province, Amazonas, Peru. This species has not been described before in Peru.

Progeny from these specimens tested for susceptibility to the BH and SJ strains of Schistosoma mansoni which under natural conditions develop in $B$. glabrata of Belo Horizonte and B. tenagophila of São José dos Campos, respectively.

Eighty specimens were exposed to the BH strain of which 13 or $16.2 \%$ developed infection with secondary sporocysts. However no elimination of cercariae was observed even in a specimen which lived 88 days after exposure. No spontaneous cure was found in this batch.

of 40 B. straminea exposed to strain SJ, 9 or $22.5 \%$ became infected but only two eliminated a few cercariae on two consecutive days at 57 and 77 days after exposure. One died and one underwent spontaneous cure. In two other infected snails in which infection wes visible through the shell spontaneous cure was noted.

Serial histological sections of 9 snails were made after individual exposure to 50 miracidia of the $\mathrm{BH}$ strain and fixed 6-120 hours after expasure. These showed sporocysts in development and invaded by amebocytes without forming granulomas in the host tissues, demonstrating the snails were susceptible.

The population studied behaved experimentally in a manner similar to other populations of B. straminea tested in the laboratory in that a low susceptibility was demonstrated. However this does not exclude the possibility that this species could maintain the parasitic cycle in its area of distribution.

\section{REFERENCIAS BIBLIOGRAFICAS}

1. BARBOSA, F.S. A note on Biomphalaria straminea (Dunker, 1848) from Manaus State of Amazonas, Brazil. Rev. Soc. Bras. Med. Trop. 2:77-78, 1968.

2. BARBOSA, F.S. \& COELHO, M.C. Qualidades de vetor dos hospedeiros de Schistosoma mansoni no nordeste do Brasil I. Suscetibilidade de A. glabratus e $T$. centimetralis à infecção por $S$. man. soni. Publ. Av. Inst. Aggeu Magalhães. 3:5562, 1954.

3. BARBOSA, F.S. \& FIGUEIREDO, T. Susceptibility of the snail intermediate hosts of Schistosomiasis from northeastern Brazil to the infection with Schisto- soma mansoni. Rev. Inst. Med. Trop. São Paulo 12 (3):198-206, 1970.

4. CHAIA, G. - Técnica para concentração de miracídios. Rev. Brasil. Malar. Doen. Trop. 8:355-357, 1956.

5. COELHO, M.V. \& BARBOSA, F.S. Qualidades de vetor dos hospedeiros de Schistosoma mansoni no nordeste do Brasil. III. Duração da infestação e eliminação de cercárias em Tropicorbis centimetralis. Pub. Av. Inst. Aggeu Magalhães. 5:21-29, 1956.

6. CORREA, L.R. \& PARAENSE, W.L. Susceptibility of Biomphalaria amazo nica to infection with two strains of 
Schistosoma mansoni. Rev. Inst. Med. Trop. São Paulo. 13 (6):387-390, 1971.

7. GERKEN, S.E., ARAUJO, M. de P. \& FREITAS, J.R. de - Suscetibilidade da Biomphalaria straminea da região de Lagoa Santa (MG) ao Schistosoma mansoni. Rev. Inst. Med. Trop. São Paulo. 17:388-343, 1975.

8. PARAENSE, W.L. \& CORREA, L.R. Susceptibility of Australorbis tenago philus to infection with Schistosoma mansoni. Rev. Inst. Med. trop. São Paulo. 5:23-29, 1963.

9. PARAENSE, W.L., IBAÑEZ, N.H. \& MIRANDA, C.H. - Australorbis tena- gophilus in Peru, and its susceptibility to Schistosoma mansoni. Am. J. Trop. Med. \& Hyg. 13:534540, 1964.

10. PARDAL, P.O., VIANNA, C.M., SANTOS, M.F. \& SOUZA, I.M. - Desativação de foco de esquistossomose no Vale do Tapajós, no Estado do Pará. Resumo. XII Congresso Soc. Brasil. Med. Trop. I Congresso Soc. Brasil Parasit., Belém, Pará, 1976.

11. WHO. PAN AMERICAN HEALTH ORGANIZATION. A Guide for the identification of the snail intermediate hosts of schistosomiasis in the Americas. Scientific Publication n? 168, 1968. 